\title{
Identification of cell cycle as the critical pathway modulated by exosome-derived microRNAs in gallbladder carcinoma
}

\author{
$\mathrm{Li} \mathrm{Su}^{1,2} \cdot$ Jicheng Zhang ${ }^{3} \cdot$ Xinglong Zhang ${ }^{3} \cdot$ Lei Zheng $^{1,2} \cdot$ Zhifa Zhu $^{1,2}$
}

Received: 23 August 2021 / Accepted: 29 September 2021 / Published online: 16 October 2021

(c) The Author(s) 2021

\begin{abstract}
Gallbladder cancer (GBC), the most common malignancy in the biliary tract, is highly lethal malignant due to seldomly specific symptoms in the early stage of GBC. This study aimed to identify exosome-derived miRNAs mediated competing endogenous RNAs (ceRNA) participant in GBC tumorigenesis. A total of 159 differentially expressed miRNAs (DEMs) was identified as exosome-derived miRNAs, contains 34 upregulated exo-DEMs and 125 downregulated exo-DEMs based on the expression profiles in GBC clinical samples downloaded from the Gene Expression Omnibus database with the R package. Among them, 2 up-regulated exo-DEMs, hsa-miR-125a-3p and hsa-miR-4647, and 5 down-regulated exo-DEMs, including hsa-miR-29c-5p, hsa-miR-145a-5p, hsa-miR-192-5p, hsa-miR-194-5p, and hsa-miR-338-3p, were associated with the survival of GBC patients. Results of the gene set enrichment analysis showed that the cell cycle-related pathways were activated in GBC tumor tissues, mainly including cell cycle, M phase, and cell cycle checkpoints. Furthermore, the dysregulated ceRNA network was constructed based on the IncRNA-miRNA-mRNA interactions using miRDB, TargetScan, miRTarBase, miRcode, and starBase v2.0., consisting of 27 lncRNAs, 6 prognostic exo-DEMs, and 176 mRNAs. Together with prognostic exo-DEMs, the STEAP3-AS1/hsa-miR-192-5p/MAD2L1 axis was identified, suggesting lncRNA STEAP3-AS1, might as a sponge of exosome-derived hsa-miR-192-5p, modulates cell cycle progression via affecting MAD2L1 expression in GBC tumorigenesis. In addition, the biological functions of genes in the ceRNA network were also annotated by Gene Ontology and Kyoto Encyclopedia of Genes and Genomes. Our study promotes exploration of the molecular mechanisms associated with tumorigenesis and provide potential targets for GBC diagnosis and treatment.
\end{abstract}

Keywords Differentially expressed genes $\cdot$ Gallbladder cancer $\cdot$ miRNA $\cdot$ ceRNA $\cdot$ Carcinogenesis

\section{Background}

Gallbladder carcinoma (GBC), the most common malignancy in the biliary tract, is highly lethal malignant [1]. Despite advances in multiple treatments including surgical

Li Su and Jicheng Zhang have contributed equally to this work.

$\mathrm{Li} \mathrm{Su}$

suli@ahmu.edu.cn

1 Department of Integrated Traditional and Western Medicine in Oncology, The First Affiliated Hospital of Anhui Medical University, Hefei 230022, People's Republic of China

2 Center of Integrated Traditional and Western Medicine in Oncology, Anhui Medical University, Hefei 230022, People's Republic of China

3 Anhui University of Traditional Chinese Medicine, Hefei 230012, People's Republic of China resection, chemotherapy, and radiotherapy, more than $90 \%$ of patients are diagnosed at the advanced stage due to seldomly specific symptoms in the early stage of GBC [2-4]. These patients cannot be effectively treated and have an extremely low survival rate consequently [5]. Therefore, it is critical to focus on the exploration of new therapeutic interventions and early prognostic markers to improve the prognoses of patients with GBC.

The regulation of competing for endogenous RNAs (ceRNAs), which are transcripts that can regulate each other at the post-transcription level by competing for shared miRNAs, is involved in the physiology and development of diseases, especially cancer initiation and progression $[6,7]$. MicroRNAs (miRNAs) are a class of small, endogenous, non-coding RNAs with a length of $\sim 22$ nucleotides, playing an important role in regulating genes associated with malignant biological behavior in cancer cells [8-11]. The abnormal expression of long-chain non-coding RNA (lncRNA), 
which acts as microRNA decoys to modulate gene expression, is correlated with the occurrence and development of tumors and other diseases [12-14]. LncRNA could inhibit miRNA function, as "sponges" of natural miRNA, by competing with the binding of the microRNA response elements (MREs) in the complex $[13,15]$. Some recent studies demonstrated that specific miRNAs are functionally involved in GBC development through modulating cell proliferation, apoptosis, migration, invasion, and metastasis, including miR-155, miR-200a, miR-182, miR-34a, and miR-130a [16-18]. Aberrant lncRNAs expression was indicative of the prognosis of GBC patients, and lncRNAs showed promise as diagnostic, prognostic, predictive biomarkers for GBC [19-21].

Exosomes, a type of extracellular vesicles (EVs) with diameters ranging from 40 to $100 \mathrm{~nm}$, are widely released from many cell types into the extracellular space. Recently, mRNAs, IncRNAs, and miRNAs have been identified in exosomes. Dysregulation of exosome-derived ncRNAs is associated with tumor invasion, migration and metastasis, and drug resistance [22-24]. However, there are no studies specifically focused on the exosome-derived ncRNAs and their regulation of ceRNA networks in GBC. In the present study, we conducted a multi-step analysis using various $\mathrm{R}$ language packages on the expression profiles of clinical samples downloaded from the Gene Expression Omnibus (GEO) database to identify the differentially expressed mRNAs and ncRNAs in the GBC. The exosome-derived miRNAs associated with GBC patients' survival were identified, and the related ceRNA network was established based on the lncRNA-miRNA-mRNA interaction. The gene set enrichment analysis (GSEA) of the differentially expressed genes regulated by exosome-derived miRNAs was identified to investigate the downstream regulation role of the exosomederived miRNAs.

\section{Materials and methods}

\section{Expression profiles information}

The datasets GSE104165 and GSE74048 were downloaded from the GEO database (https://www.ncbi.nlm.nih.gov/ geo/). The GSE104165 microarray expression profile dataset, which was based on GPL18402 ([Agilent-046064], Unrestricted_Human_miRNA_V19.0_Microarray), contained $40 \mathrm{GBC}$ with long-term $(n=20)$ and short-term $(n=20)$ survival, and 8 normal gallbladder tissues [10]. The GSE74048 microarray expression profile dataset, which was based on GPL20115 ([Agilent-067406], Human CBC IncRNA + mRNA microarray V4.0), contained 3 pairs of human GBC tissues and the matched peri-carcinomatous tissues.

\section{Differential expression analysis}

The differentially expressed genes (DEGs), lncRNAs (DELs), and miRNAs (DEMs) between GBC tissues and normal tissues of the GEO datasets, were calculated using the "limma" package with voom method in R, respectively [25]. The False Discovery Rate (FDR)—adjusted $P$ value $<0.05$ by the Benjamini-Hochberg method and $\mid \log 2$ fold change (FC)| $>2$ were set as cut-off criteria for DEMs and DELs, while as adjusted $P$ value $<0.05$ and $\mid \log 2 \mathrm{FCl}>1.5$ were for DEGs. Visualization of the identified DEGs and DEMs including volcano plot and heatmap were performed with the "ggplot2" and "pheatmap" packages of R, respectively [26]. Then, DEMs and exosome miRNAs, which were obtained from the exosome databases, ExoRBase and EVmiRNA, were intersected using the "VennDiagram" package of $\mathrm{R}$, and the overlapped genes were identified as the exo-DEMs. The GBC tissues from GSE104165 were divided into the long survival group and short survival group based on the patients' survival. Then, the expression profiles of exo-DEMs were analyzed between the two groups, and the survival-related exo-DEMs were selected for subsequent analysis.

\section{GSEA analysis of the survival-related exo-DEMs target genes}

To elucidate the molecular mechanism of the exo-DEMs associated with survival, the interactions of miRNA-target genes were obtained from the reliable online miRNA-mRNA databases, including miRDB, TargetScan, and miRTarBase, using the "multiMiR" package in R (http://multimir. org/). Interactions of miRNA-target genes were obtained based on experimental verification of luciferase reporter assay. Then, the differential expression analysis of these target genes between GBC tissues and normal tissues from GSE74048 was performed, and the possible pathways and functions were predicted using the GSEA method with the Kyoto Encyclopedia of Genes and Genomes (KEGG) and Reactome databases. NOM $P$ value $<0.05$ was considered statistically significant. The results were visualized with the "clusterProfile" package in R [27].

\section{Construction of the survival-related exo-DEMs mediated ceRNA network}

LncRNA-miRNA interaction pairs were predicted using the online databases, miRcode (http://www.mircode.org/index. php) and starBase v2.0 (http://starbase.sysu.edu.cn/starb ase2/). Then integrated with the miRNA-mRNA interactions to establish a dysregulated IncRNA-miRNA-mRNA 
ceRNA network and visualized using Cytoscape software. Furthermore, the genes and the lncRNAs in ceRNA networks were intersected with DEGs and DELs, respectively, using Venn diagram, to identify the DEGs and DELs in the ceRNA networks. The gene expression was analyzed in GBC and normal tissue using the paired Student's $t$-test. The twosided $P<0.05$ was considered statistically significant.

\section{Functional enrichment analysis and protein-protein interaction of the ceRNA network}

To better understand the biological functions of the integrated ceRNA network, Gene Ontology (GO) covering biological processes (BP), molecular functions (MF), and cellular components (CC), enrichment analyses were performed. The whole human genome was set as the background, and functional categories with adjusted $P<0.01$ were considered significant. The results were visualized with the "PerformanceAnalytics" (https://cran.r-project. org/web/packages/PerformanceAnalytics/) package of R. Furthermore, the protein interaction network was mapped with the Search Tool for the Retrieval of Interacting Genes/ Proteins (STRING) website (http://string-db.org/) and visualized using Cytoscape software [28].

\section{Results}

\section{Identification of DEMs, DELs, and DEGs in gallbladder carcinoma}

A total of 843 DEGs and 895 DELs between GBC tissues and the matched peri-carcinomatous tissues were obtained after analyzing expression profiles from GSE74048 datasets. Among them, 246 mRNAs and 716 lncRNAs were significantly upregulated; while 597 mRNAs and 179 lncRNAs were significantly downregulated, respectively. Meanwhile, 204 DEMs, including 71 up-regulated and 133 down-regulated miRNAs, between GBC tissues and normal tissues were obtained from GSE104165 dataset, respectively. The volcano plots and heatmaps illustrated the significant differences and distribution of the fold change in DEMs, DELs, and DEGs (Fig. 1a, b).

\section{The exosome DEMs associated with the GBC patients' survival}

To screen the exosome-related DEMs in GBC compared with normal tissues, Venn diagram analysis was used to obtain the intersection between DEMs and miRNAs from exosomes. Subsequently, a total of 159 overlapped DEMs were identified, containing 34 upregulated exo-DEMs and 125 downregulated exo-DEMs (Fig. 1c). Seven survival associated exo-DEMs were obtained through the differential expression analysis between the long survival group and the short survival group (Fig. 1d), including 2 up-regulated exoDEMs, hsa-miR-125a-3p and hsa-miR-4647, and 5 downregulated exo-DEMs, hsa-miR-29c-5p, hsa-miR-145a-5p, hsa-miR-192-5p, hsa-miR-194-5p, and hsa-miR-338-3p. Results showed that the 2 up-regulated exo-DEMs were up-regulated in the short survival group, while the 5 downregulated exo-DEMs were up-regulated in the long survival group (Fig. 1e).

\section{GSEA analysis of the survival-related exo-DEMs target genes}

To elucidate the molecular mechanism of the exo-DEMs with patients' survival, GSEA analysis of target genes was performed. Result showed that the GBC tumor tissues were mainly enriched in KEGG pathways including cell cycle (hsa04110, $P=0.002$ ), protein processing in endoplasmic reticulum (hsa04141, $P=0.002$ ), and carbon metabolism (hsa01200, $P=0.004$ ), while as the normal tissues were mainly enriched in proteoglycans in cancer (hsa05205, $P=0.001$ ), and serotonergic synapse (hsa04726, $P=0.004$ ) (Fig. 2a; SI 1). That indicated the genes in cell cycle, protein processing in endoplasmic reticulum, and carbon metabolism were activated in GBC tumor tissues, and the genes in pathways of proteoglycans in cancer, and serotonergic synapse were suppressed (Fig. 2b). Reactome pathway enrichment also indicated that the cell cycle-related pathways were activated in GBC tumor tissues, including cell cycle (R-HSA-1640170, $P=0.002$ ), cell cycle, mitotic (R-HSA69278, $P=0.003$ ), M phase (R-HSA-68886, $P=0.002$ ), cell cycle checkpoints (R-HSA-69620, $P=0.002$ ) (Fig. 2c, d; SI 2).

\section{Survival-related exo-DEMs mediated ceRNA network}

To better understand the biological regulation roles of the exo-DEMs with patients' survival, we constructed the dysregulated ceRNA network based on the IncRNA-miRNAmRNA interactions. The regulatory relationship between 176 mRNAs and 6 of survival-related exo-DEMs with patients' survival was found based on experimental verification of luciferase reporter assay (SI 3). Then, 27 lncRNAs were predicted to interact with the exo-DEMs using the online databases, miRcode, and starBase v2.0. Finally, integrated the relationship of miRNAs and mRNAs, miRNAs and lncRNAs, a ceRNA network consisting of 27 lncRNAs, 6 miRNAs, and 176 mRNAs was constructed (Fig. 3a). In addition, we calculated the connection degree of each gene by topology to clarify its importance in the ceRNA network. mRNA (CDH2), IncRNA (KCNQ1OT1), and miRNAs 
a
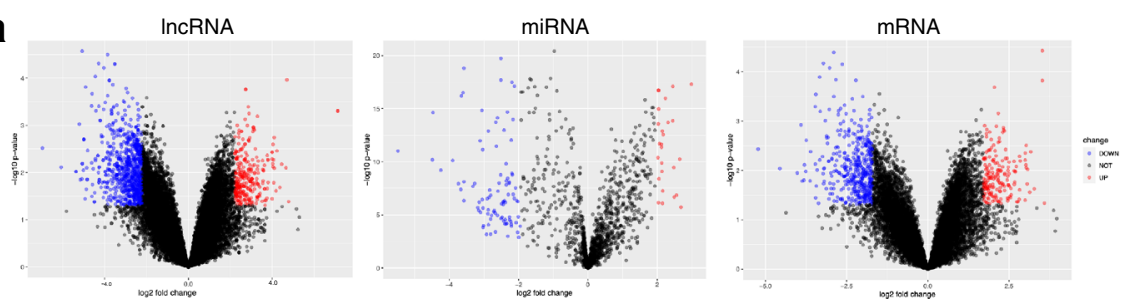

b
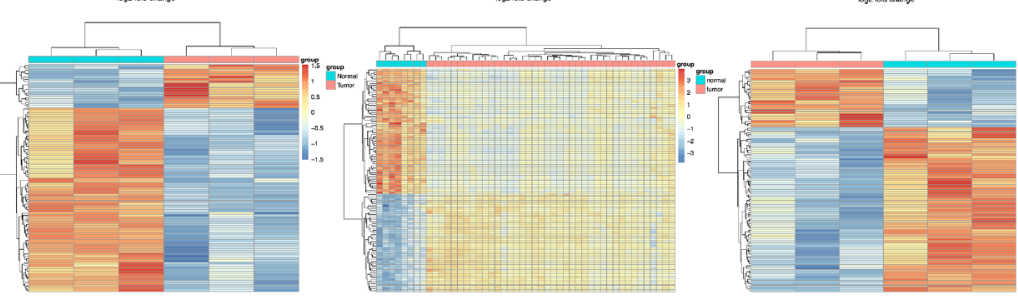

d
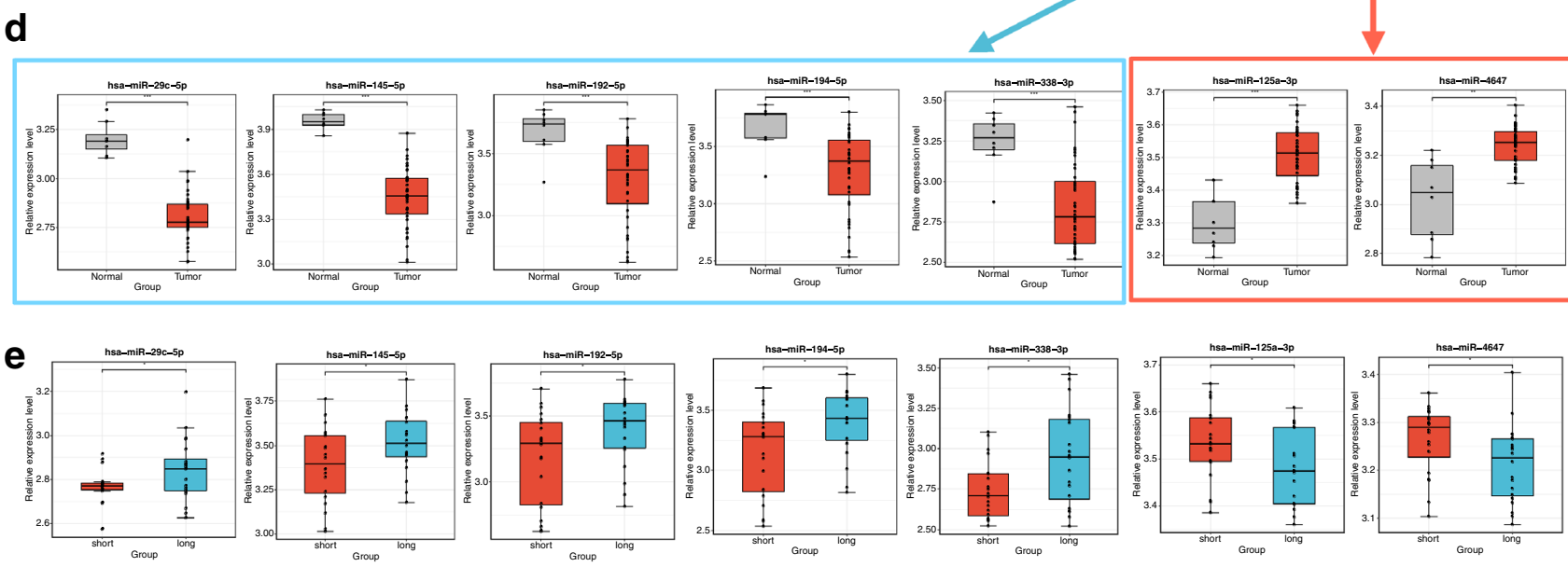

Fig. 1 Identification of DEL, exosomal DEMs and DEGs between GBC and normal tissues. a Volcano plot of DEL, DEMs, and DEGs for datasets from GEO. X-axis: $\log 2$ fold change; Y-axis: $-\log 10$ $(P$ value) for each gene; vertical-dotted lines: fold change $\geq 2$ or $\leq 2$ for DELs and DEMs, fold change $\geq 1.5$ or $\leq 1.5$ for DEGs; horizontal-dotted line: the significance cut off (adjusted $P$ value $=0.05$ ). The red dot represents up-regulated genes, and the blue dot represents down-regulated genes. b Gene expression heatmap of DEL, DEMs,

(hsa-miR-145-5p) were considered the most important genes among the lncRNAs, miRNAs, and mRNAs, respectively (SI 4).

\section{Functional enrichment and expression analysis of genes in the ceRNA network}

GO enrichment and KEGG pathway analysis were used to investigate the mechanisms associated with the ceRNA network with the threshold set as adjusted $P<0.01$. The significantly enriched GO terms were illustrated and available in Fig. 3b, c and SI 5. Most importantly, among the enriched biological processes were mainly including cellular process (GO:0009987, $P=2.96 \mathrm{E}-17)$, biological regulation (GO:0065007, $P=8.64 \mathrm{E}-22$ ), response to stimulus and DEGs, respectively. c Venn diagrams of the overlapping genes between exosome-derived miRNAs with up-regulated DEMs and down-regulated DEMs, respectively. d Box plots that represent the expression levels of exo-DEMs between tumor tissues and normal tissues. e Box plots that represent the expression levels of exo-DEMs between the tissues from long survival and short survival patients. **** indicates $P<0.0001$, *** indicates $P<0.001$, ** indicates $P<0.01$

(GO:0050896, $P=5.94 \mathrm{E}-24)$. Among the molecular functions obtained from $\mathrm{GO}$ enrichment analysis were organic cyclic compound binding (GO:0097159, $P=7.62 \mathrm{E}-10$ ), protein binding (GO:0005515, $P=3.22 \mathrm{E}-29$ ), heterocyclic compound binding (GO:1901363, $P=8.71 \mathrm{E}-10)$. The most notable significantly enriched cellular components included cell (GO:0005623, $P=4.94 \mathrm{E}-10$ ), intracellular (GO:0005622, $P=5.57 \mathrm{E}-11$ ), intracellular organelle (GO:0043229, $P=7.91 \mathrm{E}-10$ ). Meanwhile, KEGG analysis suggested that the significantly enriched pathways including Pathways in cancer (hsa05200, $P=3.48 \mathrm{E}-24$ ), Proteoglycans in cancer (hsa05205, $P=2.05 \mathrm{E}-21)$, MicroRNAs in cancer (hsa05206, $P=5.13 \mathrm{E}-24)$, Cell cycle (hsa04110, $P=1.05 \mathrm{E}-13$ ), Hepatocellular carcinoma (hsa05225, $P=4.74 \mathrm{E}-13)($ SI 6$)$. 


\section{a}
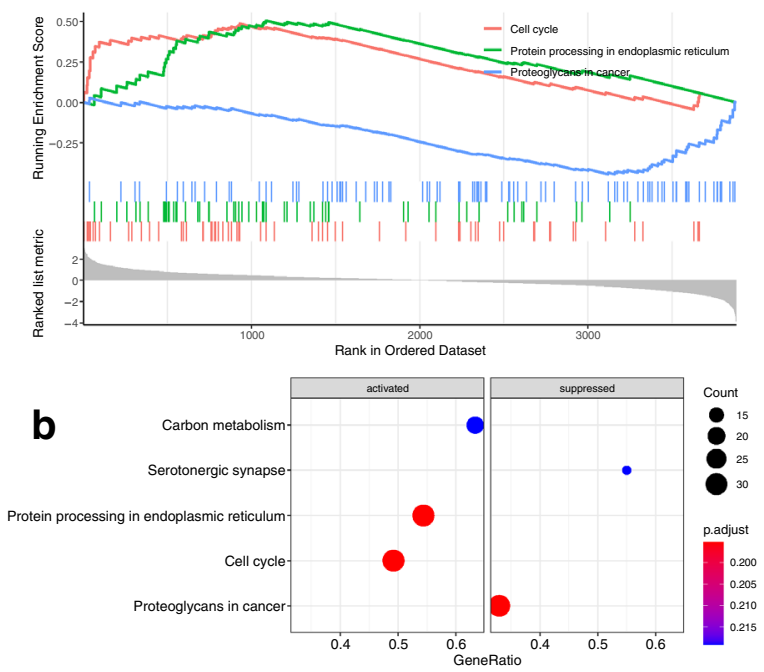

C
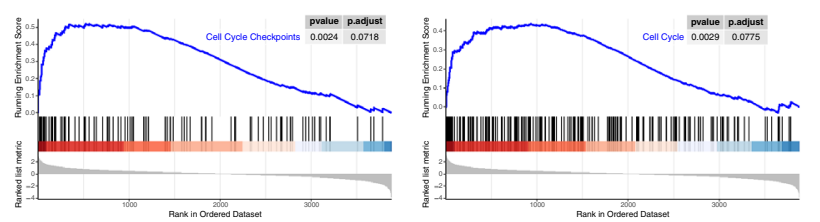

Fig. 2 GSEA analysis revealed the target genes of seven survivalrelated exo-DEMs mainly enriched in cell cycle-related pathways. a, b Illustrate the top elements significantly enriched in the KEGG

Protein-protein interaction of the gene in the ceRNA networks shown that the high-scoring proteins were obtained including CD44, MYC, CCND1, ESR1, IL6, and VEGFA, which suggesting that they might jointly regulate the occurrence and development of tumor (Fig. 4). Expression analysis indicated that five lncRNAs and ten mRNAs were differentially expressed between GBC tumor tissues and normal tissues, including seven mRNAs down-regulated in tumor tissues, while the other three mRNAs and all the five lncRNAs up-regulated in tumor tissues (Fig. 5).

\section{Cell cycle as a critical pathway in GBC tumorigenesis}

Based on the integrated ceRNA network and GSEA analysis, the STEAP3-AS1/hsa-miR-192-5p/MAD2L1 axis, which participants in cell cycle pathway, was identified as critical in GBC tumorigenesis. Besides, as shown in Fig. 6, we also found that mainly members in this pathway were differentially expressed, including 17 genes were up-regulated in GBC tumor tissues, while three genes were down-regulated. d
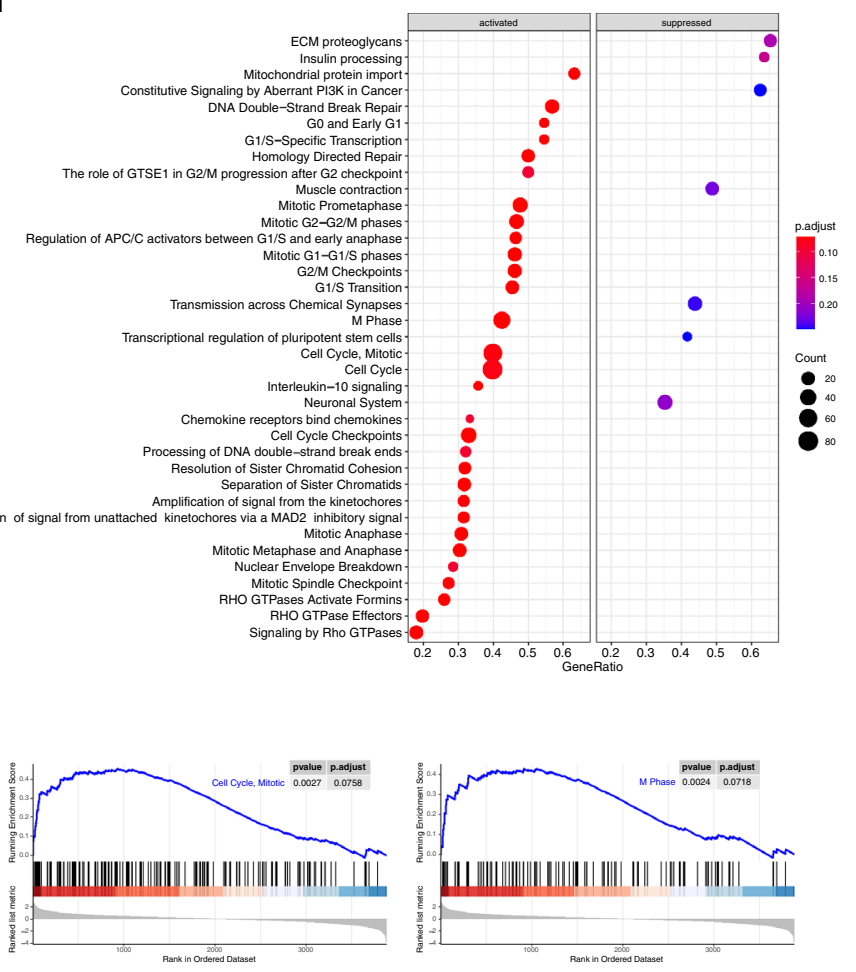

pathway with a $P$ value of less than 0.05 . c, $\mathbf{d}$ The Reactome pathway enriched in up-regulated target genes and down-regulated target genes with a $P$ value of less than 0.01 , respectively

\section{Discussion}

As dysregulation of exosomal ncRNAs has been highlighted for critical functions in tumor invasion, migration and metastasis, and drug resistance. With the purpose of identifying the exosomal miRNAs mediated ceRNA participant in GBC tumorigenesis and to investigate the potential biomarkers for better detection and therapy, we analyzed the gene expression profiles of the GEO datasets and established a lncRNA-miRNA-mRNA network mediated by prognostic exo-DEMs at the transcriptomewide level to provide a useful foundation for the regulatory function research in GBC.

We identified 204 DEMs, including 71 up-regulated and 133 down-regulated miRNAs, between GBC tissues and normal tissues. A total of 159 DEMs was identified as exosomal miRNAs, containing 34 up-regulated exo-DEMs and 125 down-regulated exo-DEMs. Seven of these exoDEMs were differentially expressed between the long-term survival group and short-term survival group of GBC patients, including 2 up-regulated hsa-miR-125a-3p and hsa-miR-4647, and 5 down-regulated hsa-miR-29c-5p, 


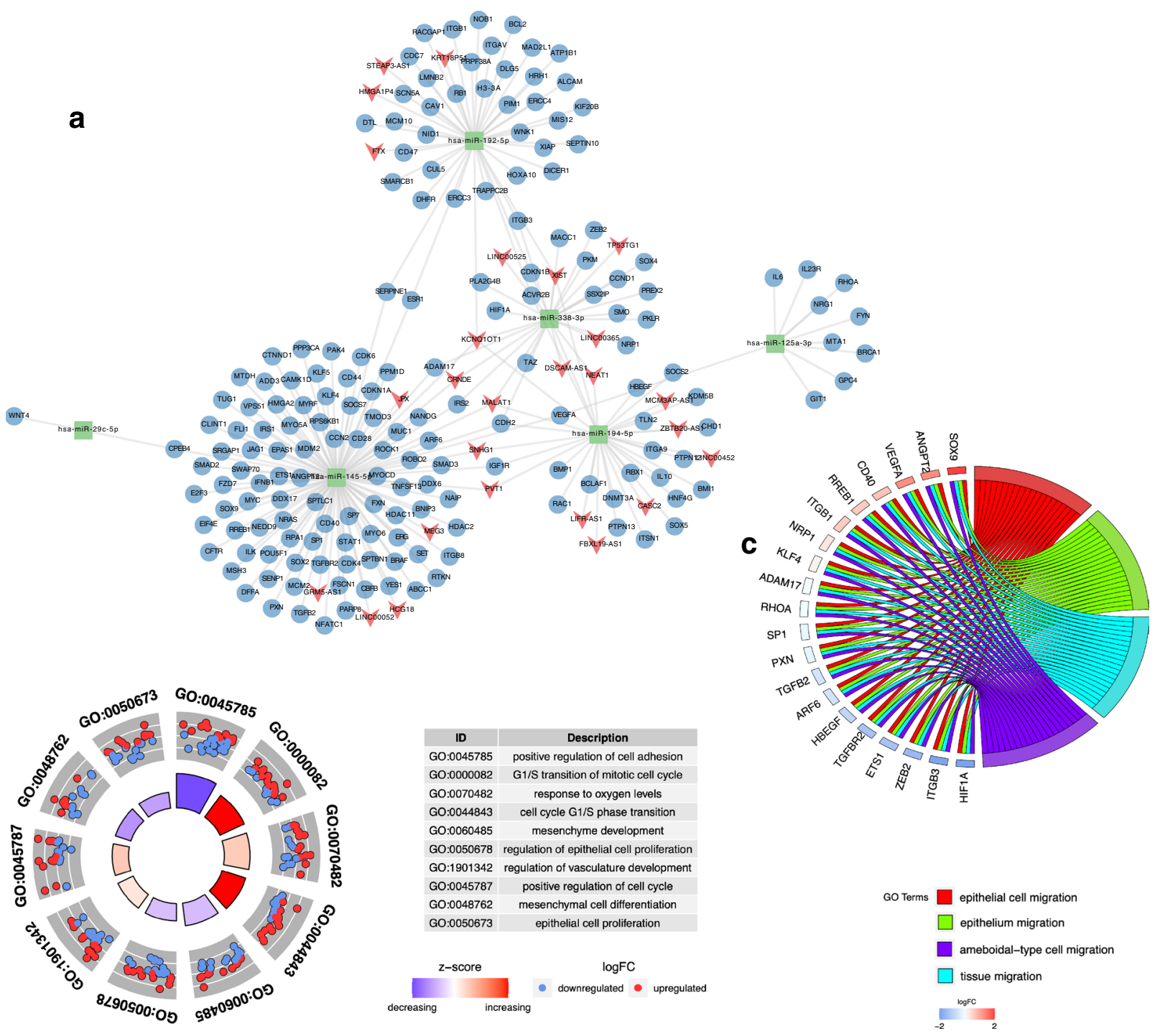

Fig. 3 The ceRNA network of survival-related exo-DEMs, lncRNAs, and mRNAs. a Green rectangles indicate exo-DEMs, red arrows indicate lncRNAs, and blue circles indicate mRNAs; $\mathbf{b}$ illustrate the top

hsa-miR-145a-5p, hsa-miR-192-5p, hsa-miR-194-5p, and hsa-miR-338-3p, indicating that they had a prognostic relationship with the survival of GBC patients. Similar results were showed in other studies in different kinds of cancers. Overexpression of hsa-miR-125a-3p significantly inhibited the proliferation and invasion of lung cancer cells, and also is readily accessible as a diagnostic biomarker for early-stage colon cancer (CRC) [29, 30]. Exosomes secreted by human adipose-derived MSCs (adMSC-Exo) can transfer miR-125a to endothelial cells and promote angiogenesis through repressing the angiogenic inhibitor delta-like 4 (DLL4) by targeting its 3' untranslated region [31]. miR-192-5p, which is associated with TRIM44, its upregulation suppressed tumor behaviors in lung cancer cells [32]. Bone marrow-derived mesenchymal stem cells
10 elements significantly enriched in the three GO categories; $\mathbf{c}$ the significant GO terms associated with the DEGs. $P$ value is less than 0.01

(BMSCs)-secreted miR-192-5p can delay the event of the inflammatory response in rheumatoid arthritis (RA) [33]. Researchers also found that human adipose mesenchymal stem cell exosomal miR-192-5p targeted IL-17RA to regulate Smad pathway in hypertrophic scar (HS) fibrosis [34]. Meanwhile, in contrast to our results, miR-192-5p was upregulated in nasopharyngeal carcinoma (NPC) tissues and was identified as "Exosomal onco-miRs" in esophageal cancer (EC). Exosomal miR-194-5p enhanced DNA damage response in the residual tumor repopulating cells to potentiate tumor repopulation $[35,36]$. miR-194-5p was also found significantly downregulated in exosomes of chronic kidney disease (CKD) patients serum [37, 38].

After that, GSEA analysis was performed to investigate the molecular mechanism of the prognostic exo-DEMs. 


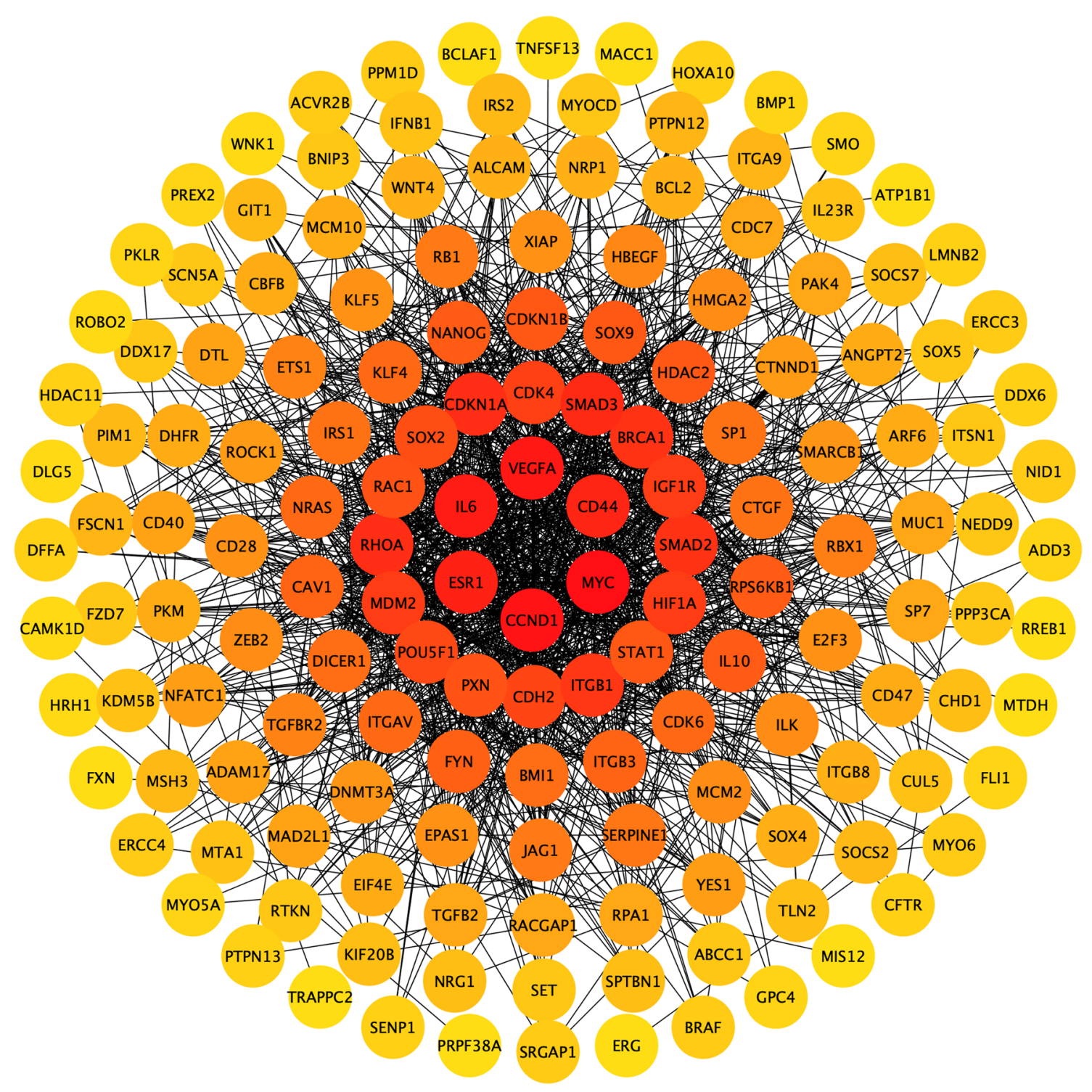

Fig. 4 Protein-protein interaction network displaying the interactions among the genes in the ceRNA networks, as determined with STRING and illustrated in Cytoscape

Results indicated that the enriched KEGG and Reactome pathways which activated in GBC tumor tissues were mainly associated with the cell cycle-related pathways including cell cycle, $\mathrm{M}$ phase, and cell cycle checkpoints. Furthermore, the dysregulated ceRNA network based on the IncRNA-miRNA-mRNA interactions was constructed, consisting of 27 lncRNAs, 6 prognostic exo-DEMs, and 176 mRNAs. GO enrichment and KEGG pathway analysis also showed that the genes of the ceRNA network were mainly enriched in cell cycle-related GO terms and KEGG pathways. Before the reproductive cycle of replicating DNA, cells enter a phase called G1 during which they release plenty of signals that contribute to cell division and cell fate. miRNAs regulate genes involved in checkpoints, DNA repair, and cell cycle. Dysregulated miRNAs can lead to the loss of cell cycle regulation and finally contribute to pathological situations, such as cancer [38-40]. Expression analysis indicated that 5 lncRNAs and 10 mRNAs in the ceRNA network were differentially expressed between GBC tumor tissues and normal tissues. Together with prognostic exo-DEMs, the STEAP3-AS1/ hsa-miR-192-5p/MAD2L1 axis was identified. Due to the main members in the cell cycle pathway were differentially expressed including MAD2L1, we speculated that the STEAP3-AS1/hsa-miR-192-5p/MAD2L1 axis was critical in GBC tumorigenesis through regulation of cell cycle. A recent study has found that STEAP3-AS1 downregulation could increase the expression of cyclin-dependent kinase 

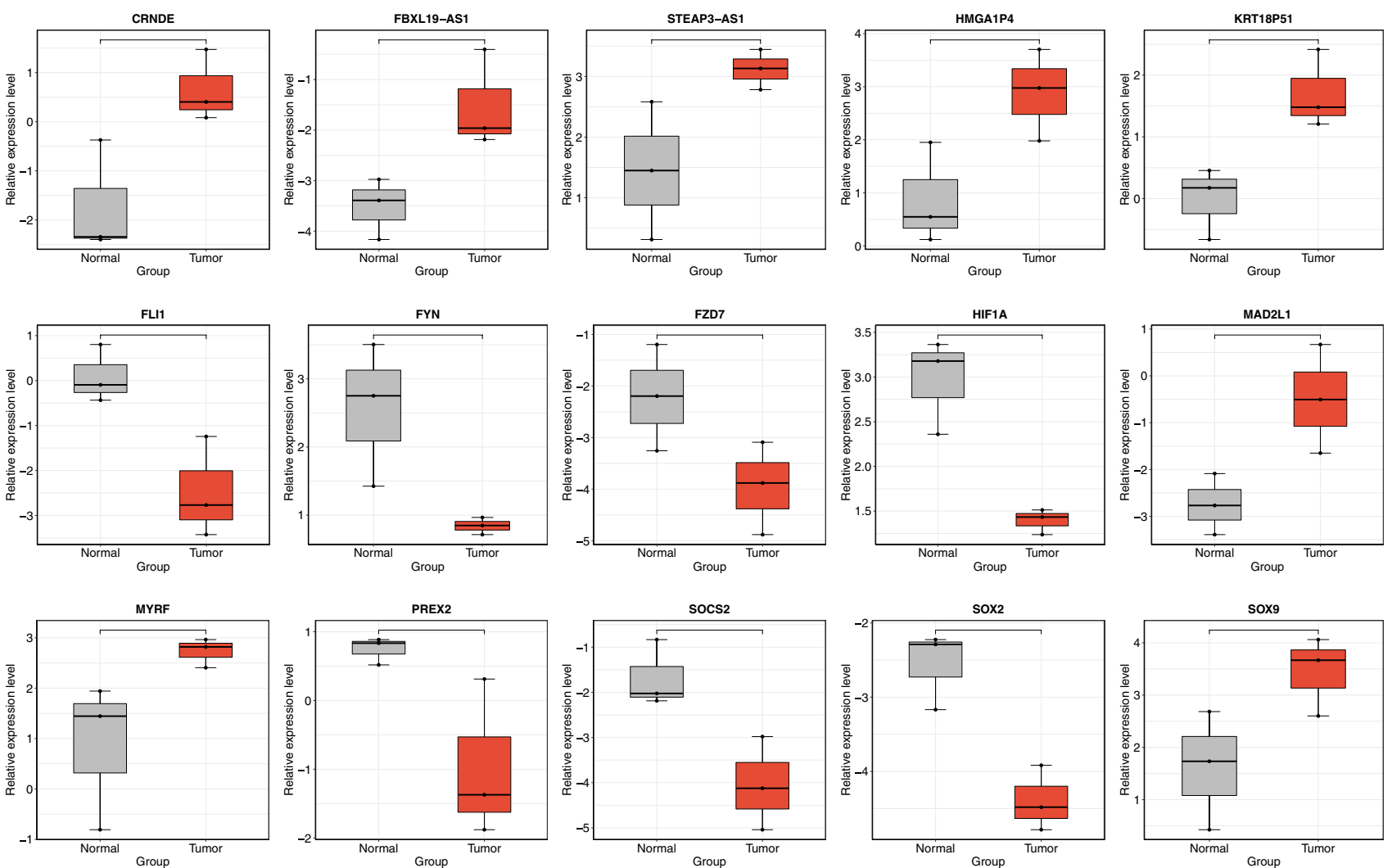

Fig. 5 Box plots that represent the differentially expression of mRNAs and lncRNAs in the ceRNA networks between tumor tissues and normal tissues. **** indicates $P<0.0001$, *** indicates $P<0.001$, ** indicates $P<0.01$

inhibitor 1C (CDKN1C) by STEAP3 upregulation and modulate the cell cycle progression in CRC [41]. STEAP3AS1 was also identified as a novel, potential prognostic biomarker, and therapeutic target for tongue squamous cell carcinoma (TSCC) [42]. STEAP3-AS1, together with HOTAIR and SOX21-AS1, as the exo-lncRNA signature, was proven to be an independent prognostic factor in glioblastoma (GBM) [43]. MAD2L1, MAD2 mitotic arrest deficient-like 1, is a critical mitotic checkpoint gene. Overexpressing MAD2L1 has shown the effect on cancer cell proliferation, migration, invasion, and cell cycle arrest [44-46]. Activation of miR-192/215 by p53 includes a set of genes which regulate DNA synthesis and G1 and G2 checkpoints, including CDC7, MAD2L1, and CUL5, to induce cell arrest and reduce tumor cell growth [47-50]. In our study, we found that STEAP3-AS1 and MAD2L1 were up-regulated in GBC tumor tissues, while as hsamiR-192-5p was down-regulated, suggesting lncRNA STEAP3-AS1, might as a sponge of exosome-derived hsamiR-192-5p, modulates cell cycle progression via affecting MAD2L1 expression in GBC tumorigenesis. Further experimental studies are required to verify this hypothesis. These findings suggest that the ceRNA network have significant effects on the pathogenesis and prognosis of GBC.

\section{Conclusion}

This study provides a better understanding of the lncRNAmiRNA-mRNA regulatory mechanism in GBC mediated by exosome-derived miRNAs. In future research, we will explore the prognostic genes and their potential function of the ceRNA axis based on the present study. 


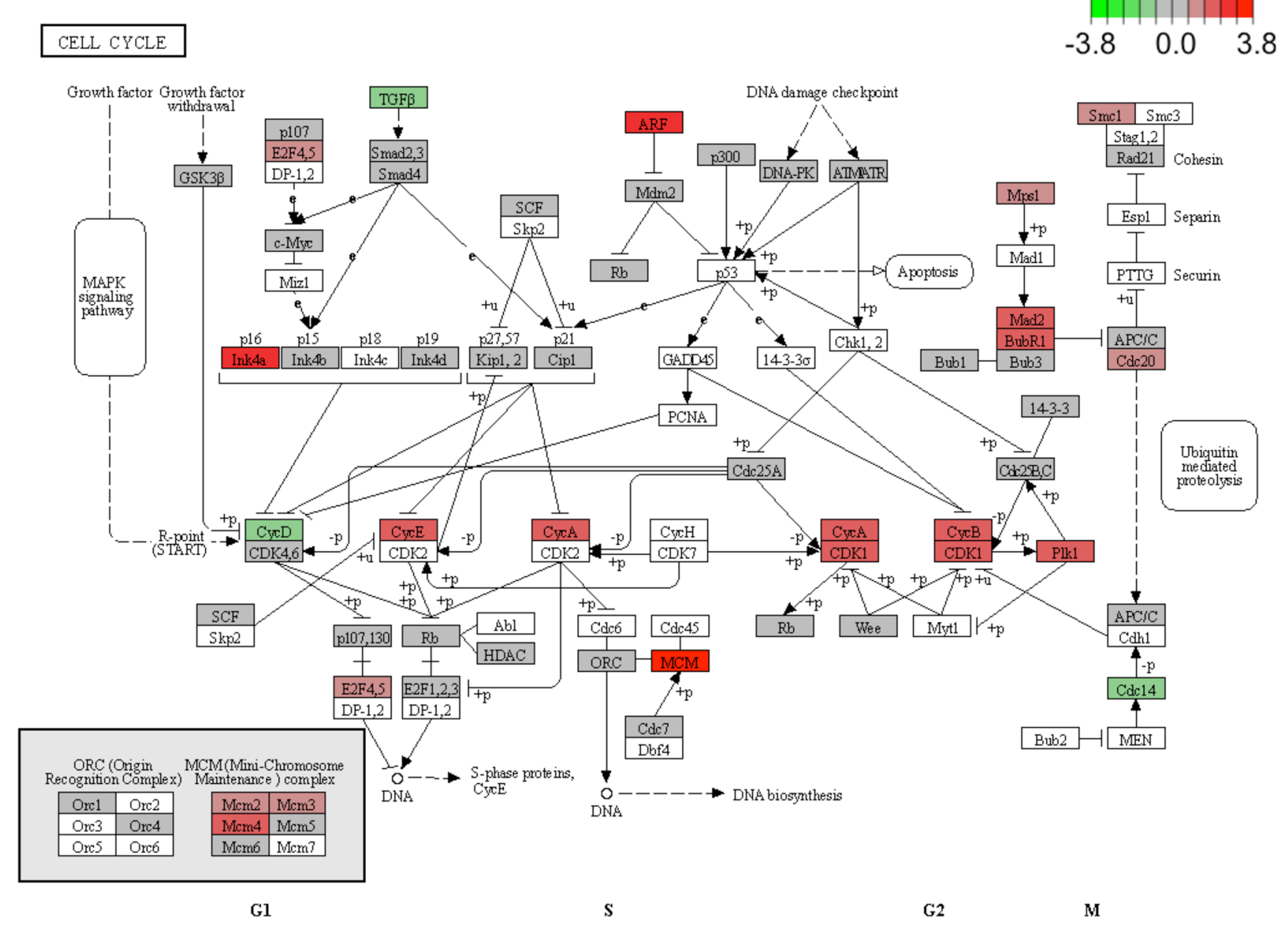

Fig. 6 Differential expression of genes associated in the cell cycle pathway between tumor and normal tissues. The red color represents up-regulated genes, the green color represents down-regulated genes, and the gray color represents non-significantly expressed genes

Supplementary Information The online version contains supplementary material available at https://doi.org/10.1007/s12032-021-01594-8.

Acknowledgements Thanks to all the peer reviewers and editors for their opinions and suggestions.

Funding Not applicable.

Data availability GEO belongs to public database. The patients involved in the database have obtained ethical approval. Users can download relevant data for free for research and publish relevant articles. Our study is based on open-source data, so there are no ethical issues and other conflicts of interest.

\section{Declarations}

Conflict of interest The authors declare that they have no competing interests.

Ethical approval Not applicable.
Open Access This article is licensed under a Creative Commons Attribution 4.0 International License, which permits use, sharing, adaptation, distribution and reproduction in any medium or format, as long as you give appropriate credit to the original author(s) and the source, provide a link to the Creative Commons licence, and indicate if changes were made. The images or other third party material in this article are included in the article's Creative Commons licence, unless indicated otherwise in a credit line to the material. If material is not included in the article's Creative Commons licence and your intended use is not permitted by statutory regulation or exceeds the permitted use, you will need to obtain permission directly from the copyright holder. To view a copy of this licence, visit http://creativecommons.org/licenses/by/4.0/.

\section{References}

1. Biswas PK. Carcinoma gallbladder. Mymensingh Med J. 2010;19:477-81.

2. Hundal R, Shaffer EA. Gallbladder cancer: epidemiology and outcome. Clin Epidemiol. 2014;6:99-109.

Consent for publication Not applicable. 
3. Yadav R, Jain D, Mathur SR, Sharma A, Iyer VK. Gallbladder carcinoma: an attempt of WHO histological classification on fine needle aspiration material. Cytojournal. 2013;10:12.

4. Ouchi K, Sugawara T, Ono H, Fujiya T, Kamiyama Y, Kakugawa $\mathrm{Y}$, et al. Diagnostic capability and rational resectional surgery for early gallbladder cancer. Hepatogastroenterology. 1999;46:1557-60.

5. Hawkins WG, DeMatteo RP, Jarnagin WR, Ben-Porat L, Blumgart LH, Fong Y. Jaundice predicts advanced disease and early mortality in patients with gallbladder cancer. Ann Surg Oncol. 2004;11:310-5.

6. Karreth FA, Pandolfi PP. ceRNA cross-talk in cancer: when cebling rivalries go awry. Cancer Discov. 2013;3:1113-21.

7. Qi X, Zhang D-H, Wu N, Xiao J-H, Wang X, Ma W. ceRNA in cancer: possible functions and clinical implications. J Med Genet. 2015;52:710-8.

8. Acunzo M, Romano G, Wernicke D, Croce CM. MicroRNA and cancer-a brief overview. Adv Biol Regul. 2015;57:1-9.

9. Wang N, Xia S, Chen K, Xiang X, Zhu A. Genetic alteration regulated by microRNAs in biliary tract cancers. Crit Rev Oncol Hematol. 2015;96:262-73.

10. Goeppert B, Truckenmueller F, Ori A, Fritz V, Albrecht T, Fraas A, et al. Profiling of gallbladder carcinoma reveals distinct miRNA profiles and activation of STAT1 by the tumor suppressive miRNA-145-5p. Sci Rep. 2019;9:4796.

11. Shin VY, Chu K-M. MiRNA as potential biomarkers and therapeutic targets for gastric cancer. World J Gastroenterol. 2014;20:10432-9.

12. Braga EA, Fridman MV, Moscovtsev AA, Filippova EA, Dmitriev AA, Kushlinskii NE. LncRNAs in ovarian cancer progression, metastasis, and main pathways: ceRNA and alternative mechanisms. Int J Mol Sci. 2020;21:8855.

13. Kong X, Duan Y, Sang Y, Li Y, Zhang H, Liang Y, et al. LncRNACDC6 promotes breast cancer progression and function as ceRNA to target CDC6 by sponging microRNA-215. J Cell Physiol. 2019;234:9105-17.

14. Sui J, Li Y-H, Zhang Y-Q, Li C-Y, Shen X, Yao W-Z, et al. Integrated analysis of long non-coding RNA-associated ceRNA network reveals potential lncRNA biomarkers in human lung adenocarcinoma. Int J Oncol Spandidos Publ. 2016;49:2023-36.

15. Xiao B, Zhang W, Chen L, Hang J, Wang L, Zhang R, et al. Analysis of the miRNA-mRNA-lncRNA network in human estrogen receptor-positive and estrogen receptor-negative breast cancer based on TCGA data. Gene. 2018;658:28-35.

16. Gupta A, Sharma A, Yadav A, Rastogi N, Agrawal S, Kumar A, et al. Evaluation of miR-27a, miR-181a, and miR-570 genetic variants with gallbladder cancer susceptibility and treatment outcome in a North Indian population. Mol Diagn Ther. 2015;19:317-27.

17. Li Z, Yu X, Shen J, Law PTY, Chan MTV, Wu WKK. MicroRNA expression and its implications for diagnosis and therapy of gallbladder cancer. Oncotarget. 2015;6:13914-21.

18. Ye Y-Y, Mei J-W, Xiang S-S, Li H-F, Ma Q, Song X-L, et al. MicroRNA-30a-5p inhibits gallbladder cancer cell proliferation, migration and metastasis by targeting E2F7. Cell Death Dis. 2018;9:410.

19. Tekcham DS, Tiwari PK. Non-coding RNAs as emerging molecular targets of gallbladder cancer. Gene. 2016;588:79-85.

20. Chen B, Li Y, He Y, Xue C, Xu F. The emerging roles of long non-coding RNA in gallbladder cancer tumorigenesis. Cancer Biomark. 2018;22:359-66.

21. Khandelwal A, Malhotra A, Jain M, Vasquez KM, Jain A. The emerging role of long non-coding RNA in gallbladder cancer pathogenesis. Biochimie. 2017;132:152-60.

22. Hu C, Meiners S, Lukas C, Stathopoulos GT, Chen J. Role of exosomal microRNAs in lung cancer biology and clinical applications. Cell Prolif. 2020;53:e12828.
23. Sun Z, Shi K, Yang S, Liu J, Zhou Q, Wang G, et al. Effect of exosomal miRNA on cancer biology and clinical applications. Mol Cancer. 2018;17:147.

24. Zhang J, Li S, Li L, Li M, Guo C, Yao J, et al. Exosome and exosomal microRNA: trafficking, sorting, and function. Genomics Proteomics Bioinform. 2015;13:17-24.

25. Law CW, Chen Y, Shi W, Smyth GK. Voom: precision weights unlock linear model analysis tools for RNA-seq read counts. Genome Biol. 2014;15:R29.

26. Ito K, Murphy D. Application of ggplot2 to pharmacometric graphics. CPT Pharmacomet Syst Pharmacol. 2013;2:e79.

27. Yu G, Wang L-G, Han Y, He Q-Y. clusterProfiler: an R package for comparing biological themes among gene clusters. OMICS. 2012;16:284-7.

28. Szklarczyk D, Gable AL, Lyon D, Junge A, Wyder S, HuertaCepas J, et al. STRING v11: protein-protein association networks with increased coverage, supporting functional discovery in genome-wide experimental datasets. Nucleic Acids Res. 2019;47:D607-13.

29. Li S, Li X, Zhao H, Gao M, Wang F, Li W. Overexpression of microRNA-125a-3p effectively inhibits the cell growth and invasion of lung cancer cells by regulating the mouse double minute 2 homolog/p53 signaling pathway. Mol Med Rep. 2015;12:5482-6.

30. Wang J, Yan F, Zhao Q, Zhan F, Wang R, Wang L, et al. Circulating exosomal miR-125a-3p as a novel biomarker for early-stage colon cancer. Sci Rep. 2017;7:4150.

31. Liang X, Zhang L, Wang S, Han Q, Zhao RC. Exosomes secreted by mesenchymal stem cells promote endothelial cell angiogenesis by transferring miR-125a. J Cell Sci. 2016;129:2182-9.

32. Zou P, Zhu M, Lian C, Wang J, Chen Z, Zhang X, et al. miR$192-5 p$ suppresses the progression of lung cancer bone metastasis by targeting TRIM44. Sci Rep. 2019;9:19619.

33. Zheng J, Zhu L, Iok In I, Chen Y, Jia N, Zhu W. Bone marrowderived mesenchymal stem cells-secreted exosomal microRNA192-5p delays inflammatory response in rheumatoid arthritis. Int Immunopharmacol. 2020;78:105985.

34. Li Y, Zhang J, Shi J, Liu K, Wang X, Jia Y, et al. Exosomes derived from human adipose mesenchymal stem cells attenuate hypertrophic scar fibrosis by miR-192-5p/IL-17RA/Smad axis. Stem Cell Res Ther. 2021;12:221.

35. Jiang M-J, Chen Y-Y, Dai J-J, Gu D-N, Mei Z, Liu F-R, et al. Dying tumor cell-derived exosomal miR-194-5p potentiates survival and repopulation of tumor repopulating cells upon radiotherapy in pancreatic cancer. Mol Cancer. 2020;19:68.

36. Liu X, Liu S, Luo D, Huang S, Wang F, Zhang B, et al. Involvement of circulating exosomal microRNAs in Jian-Pi-Yi-Shen formula protection against adenine-induced chronic kidney disease. Front Pharmacol. 2020;11:622658.

37. Magayr TA, Song X, Streets AJ, Vergoz L, Chang L, Valluru MK, et al. Global microRNA profiling in human urinary exosomes reveals novel disease biomarkers and cellular pathways for autosomal dominant polycystic kidney disease. Kidney Int. 2020;98:420-35.

38. Massagué J. G1 cell-cycle control and cancer. Nature. 2004;432:298-306.

39. Mullany LE, Herrick JS, Sakoda LC, Samowitz W, Stevens JR, Wolff RK, et al. miRNA involvement in cell cycle regulation in colorectal cancer cases. Genes Cancer. 2018;9:53-65.

40. Mens MMJ, Ghanbari M. Cell cycle regulation of stem cells by microRNAs. Stem Cell Rev Rep. 2018;14:309-22.

41. Na H, Li X, Zhang X, Xu Y, Sun Y, Cui J, et al. lncRNA STEAP3AS1 modulates cell cycle progression via affecting CDKN1C expression through STEAP3 in colon cancer. Mol Ther Nucleic Acids. 2020;21:480-91. 
42. Zhang S, Cao R, Li Q, Yao M, Chen Y, Zhou H. Comprehensive analysis of lncRNA-associated competing endogenous RNA network in tongue squamous cell carcinoma. PeerJ. 2019;7:e6397.

43. Wang Z, Ji X, Gao L, Guo X, Lian W, Deng K, et al. Comprehensive in silico analysis of a novel serum exosome-derived competitive endogenous RNA network for constructing a prognostic model for glioblastoma. Front Oncol. 2021;11:553594.

44. Foijer F, Albacker LA, Bakker B, Spierings DC, Yue Y, Xie SZ, et al. Deletion of the MAD2L1 spindle assembly checkpoint gene is tolerated in mouse models of acute T-cell lymphoma and hepatocellular carcinoma. Elife. 2017. https://doi.org/10.7554/eLife. 20873.

45. Li Y, Bai W, Zhang J. MiR-200c-5p suppresses proliferation and metastasis of human hepatocellular carcinoma (HCC) via suppressing MAD2L1. Biomed Pharmacother. 2017;92:1038-44.

46. Li J, He X, Wu X, Liu X, Huang Y, Gong Y. miR-139-5p inhibits lung adenocarcinoma cell proliferation, migration, and invasion by targeting MAD2L1. Comput Math Methods Med. 2020;2020:2953598.
47. Wang Y, Wang F, He J, Du J, Zhang H, Shi H, et al. miR-30a-3p targets MAD2L1 and regulates proliferation of gastric cancer cells. Onco Targets Ther. 2019;12:11313-24.

48. Georges SA, Biery MC, Kim S-Y, Schelter JM, Guo J, Chang $\mathrm{AN}$, et al. Coordinated regulation of cell cycle transcripts by p53-inducible microRNAs, miR-192 and miR-215. Cancer Res. 2008;68:10105-12.

49. Feng Z, Zhang C, Wu R, Hu W. Tumor suppressor p53 meets microRNAs. J Mol Cell Biol. 2011;3:44-50.

50. Mao A, Liu Y, Zhang H, Di C, Sun C. microRNA expression and biogenesis in cellular response to ionizing radiation. DNA Cell Biol. 2014;33:667-79.

Publisher's Note Springer Nature remains neutral with regard to jurisdictional claims in published maps and institutional affiliations. 